\title{
STUDIES ON THE BIOLOGY OF AND IMMUNITY AGAINST THE BACILLUS OF LEPROSY
}

\author{
WITH a CONSIDERATION OF THE POSSIBILITY OF SPECIFIC TREATMENT
}

AND PROPHYLAXIS *

CHARLES W. DUVAL, M.D., AND FRASER B. GURD, M.D.
NEW ORLEANS

Since an earlier communication ${ }^{1}$ on the cultivation of Bacillus leprce and the experimental production of leprosy by one of us (Duval) considerable light has been thrown on further study into the biology of the organism, especially with regard to its pathogenicity and viability, properties little understood prior to the cultivation of the bacillus. In the present paper we propose to give briefly our researches in this particular phase of the subject together with the results of our studies in immunity.

Soon after the cultivation of the specific organism the possibility of a serum therapy for the treatment of leprosy presented itself, and with this in view we set about to study the blood of lepers in the hope that something might accrue which would aid in subsequent work on the artificial production of an immune serum. Before beginning the work along this line it was thought advisable first to determine whether in cases of human leprosy specific antibodies are present, and if so, to find out as far as possible their nature and the mechanism of production.

\section{PATHOGENICITY}

Heretofore our attempts to infect or produce lesions in animals other than the Japanese dancing mouse have been unsuccessful; but of late we have succeeded in producing the lesion in white mice and monkeys, not only with the infested tissue from human leprosy, but with cultures. One strain of $B$. lepro in particular readily gives rise to fatal infection in the white mouse. The success with this strain has led us to test again the infectibility for the white mouse of four strains isolated more than a year ago. To our surprise, one of these (Culture II), with which we failed in former experiments to infect the white mouse, now proves infectious after more than fourteen months under cultivation, producing multiple lesions following intraperitoneal injection. The failure before

*From the Laboratories of Pathology, Tulane University, New Orleans.

I. Duval, C. W.: The Cultivation of the Leprosy Bacillus and the Experimental Production of Leprosy in the Japanese Dancing Mouse, Jour. Exper. Med., 1910, xii, 649. 
to infect this species must be attributed to some error, for it cannot be conceived that a culture would alter in this particular feature under conditions of artificial cultivation. One is led to infer, however, from the present behavior of the culture, that the infectivity of some strains of $B$. leproe, at least, are not appreciably altered by a year's sojourn outside of the animal body. It may be stated that not all strains are capable of infecting either dancing or white mice.

Thus far the experimental work would indicate that there is a considerable range in infectibility for cultures with respect to these animals; and the same is true for the bacilli used directly from the human tissues. An illustration of this variation has been observed in a rather remarkable stem that was recently isolated from the necrotic tissues of an acute case of human leprosy which had developed numerous soft subcutaneous leprous masses and had had repeated attacks of leprous fever. The initial growth resulted directly from bits of the transferred necrotic material on tryptophan agar. The acid-fast bacilli in the necrotic tissue were in enormous numbers and uncontaminated by other organisms as was subsequently proved by cultural tests.

A series of animals including four each of rats and white and Japanese dancing mice were inoculated, some subcutaneously and others intraperitoneally, with small quantities of the grumous material ( 0.5 c.c. emulsified in 1 c.c. of normal saline solution). The two white mice which received intraperitoneal injection died fourteen days after the inoculation. At autopsy both showed a general infection of the peritoneum with a pure growth of $B$. leprce, while the mesentery, omentum, visceral and parietal peritoneum contained numerous minute, firm grayish white nodules that on microscopic section proved to be typical leprous lesions. The most surprising feature was the occurrence of a slightly turbid semigelatinous peritoneal exudate which microscopically consisted almost entirely of large mononucleated cells (macrophages). Great numbers of these cells were filled with acid-fast bacilli, and scarcely any were found that did not contain a few. A pure culture of $B$. lepras was recovered from the exudate on a variety of special media, including tryptophan and glycerinated blood-agar.

The ability of $B$. leproe to cause a fatal infection in so short a period, and to induce the production of a peritoneal exudate is a new rôle for the organism in our experience with the eight stems now under cultivation. At first we were inclined to believe that death in the case of these animals had resulted from the liberation of large quantities of toxins introduced with the necrotic material and augmented by the liberation of more toxic bodies due to lytic action on the injected bacilli. This hypothesis, however, is hardly tenable if we consider the length of time that elapsed between the inoculation and death of the animal (fourteen 
days), and the fact that the bacilli had increased in number within the peritoneum and were cultivated from the exudate.

The rats which received injections of the necrotic material have not as yet developed any signs of infection; nor have the Japanese dancing mice which were injected subcutaneously evinced any evidence of the disease. In two of the Japanese mice, however, which were sacrificed four weeks after the inoculation, small 1-mm. leprous nodules were found in the spleen, liver and inguinal lymph-nodes. Unfortunately, no Japanese mice were inoculated intraperitoneally with the bacilli of the necrotic tissue.

Another series of white and dancing mice were injected in a similar manner with the artificial culture obtained from this case, four standard lonps of a heavy homogeneous suspension of bacilli being used. All the animals which received intraperitoneal injection became infected, the white mice dying one in three weeks and the other in twenty-eight days. At autopsy a semigelatinous exudate partially filled the peritoneum. In the Japanese mice, though numerous minute lesions studded the peritoneum, there was no evidence of exudate.

With regard to dancing mice, though lesions almost invariably develop after intraperitoneal injections of $B$. lepro, none of the animals die as a result, although some of them have now been under observation for more than eight months. The mice that have been sacrificed, however, present small macroscopic lesions in various organs of the body. In some instances, the mice killed five months afterward have shown no demonstrable lesions; presumably in these animals the bacilli were destroyed and the lesions in consequence healed. This assumption is based on the fact that all dancing mice killed four to ten weeks after inoculation have shown small well-defined leprous lesions. Experimentation with these animals has proved that no intermediate host is necessary in the transmission of the disease. Attempts to infect rats, rabbits and guinea-pigs have so far given negative results.

The culture which produces fatal infection in white mice is now being used in the attempt to infect larger animals, more especially the Macacus rhesus monkey. In one animal of this species which has received at weekly intervals large subcutaneous doses of the bacilli have developed at the various points of inoculation firm subcutaneous nodules many of which have increased in size. The blood of this animal shows specific agglutinins and amboceptors in the presence of the culture antigen.

With the exception of two cultures (one isolated in December, 1909, and the other in October, 1910) B. lepro, in our experience, has failed to give rise to a fatal infection in any of the laboratory animals employed. Many of the animals show multiple lesions after from four to eight weeks, but the lesions are small and tend to retrogress after a period of several months. 
The experimental lesions of leprosy are histologically identical with those in the human tissue. Although to the naked eye they appear as tubercles, they are more minute and circumscribed than the lesions of tuberculosis and do not caseate. It cannot be said, however, that they are indistinguishable from early miliary tubercles, but one accustomed to seeing the experimental leprous tubercle has very little trouble in recognizing even macroscopic differences and on microscopic examination any previously existing doubt is at once dispelled.

Though at present we are not prepared to discuss in extenso the toxic body of $B$. leprce it may be stated that experiments show apparently the presence of a soluble toxin. Four hundred million leprosy bacilli treated with 2 c.c. of a 5 per cent. glycerin solution and centrifugalized yield an extract which gives a well-marked cutaneous reaction in doses of 0.4 c.c. at the end of twenty-four hours.

\section{VIABILITY}

The question of the viability of $B$. lepres outside of the animal body is of the greatest practical importance from the standpoint of sanitation and preventive medicine. The fact that $B$. leprex cultures will remain alive and virulent for months under the most unfavorable conditions would explain much heretofore not understood about the transmission of the disease.

The bacilli can be readily cultivated from bits of infested leprous tissue which have been kept in salt solution at room temperature for more than eight months. Again, they live and retain their virulence in culture associated with other bacteria for more than a year when precaution is taken to avoid against drying of the medium. Fresh growth can also be obtained from cultures which are more than a year old, whether kept at $10 \mathrm{C}, 32 \mathrm{C}$. or $3 \% \mathrm{C}$. Even in material overgrown with saprophytic bacteria, the lepra bacilli remain viable for months and will multiply when separated from these and transferred to a suitable medium. There is no evidence to show that the ordinary pyogenic or saprophytic bacteria interfere with the longevity of $B$. lepro; as a matter of fact, the organism seems to live and flourish best in symbiosis. Where the contaminator is a non-spore-bearer it is an easy task to rid it from the culture by heating at $60 \mathrm{C}$. for one hour. In order to obtain a pure growth of $B$. leprce from a culture contaminated with saprophytic spore-bearers, the mixture may be injected intraperitoneally into a mouse, the animal killed in ten days to two weeks, and the acid-fast bacillus recovered from the peritoneum.

The leprosy bacilli will multiply slowly in transplanted bits of tissue kept at $25 \mathrm{C}$. We have found that the temperature conditions in which the bacillus will multiply range between $25 \mathrm{C}$. to $39 \mathrm{C}$, the optimum being about $32 \mathrm{C}$. In a previous paper it was stated that with the four 
strains then under cultivation growth would not occur at $37 \mathrm{C}$. We wish to correct this statement, as recent work with cultures shows that multiplication does occur at body temperature, though the best growth is obtained at from 32 to $35 \mathrm{C}$.

In our experience cultures will withstand higher degrees of temperature and for a longer period of time than any of the better-known acidfast bacilli, growth resulting after heating the bacilli for thirty minutes at $70 \mathrm{C}$. or at $65 \mathrm{C}$. for one hour. Heating at these temperatures apparently does not alter the infectivity of the bacilli for mice. Whether the organism's resistance to high temperature is due to its fatty envelope, as in the case of the tubercle bacillus, or to spores, is still undetermined. We do not doubt that the fatty substance in and about the organism protects it to a certain extent against moderate degrees of heat, but this in itself would not explain the resistance shown by some cultures. These higher resistant cultures possess "clear spaces" and deeply staining bodies within the bacilli, which in many respects are morphologically similar to the ordinary bacterial spore. That certain cultures of $B$. leprce, more especially those containing these "clear spaces," resist higher temperatures than cultures that do not contain them, has been repeatedly demonstrated by us. The true nature of these bodies is, however, still problematic and work is now in progress to determine whether or not $B$. leprce under certain conditions forms spores.

\section{CULTIVATION}

The initial growth of $B$. leproe from the human tissue on an artificial medium is in all cases obtained with considerable difficulty. The bacilli outside of the animal body multiply more slowly in the initial transplants than do bovine tubercle bacilli, and it is necessary to transfer large quantities of the macerated tissue to the culture medium unless aminoacids are used in order to insure growth, which at best is feeble and not visible macroscopically before several weeks. Again, transplantation of large numbers of bacilli from the tissues is necessary, because not onethird of the planted bacilli will multiply. The rods do not differ in size and shape from the parent organisms and grow in dense clusters, which are broken up with great difficulty. In some instances, multiplication can be accelerated by rubbing up the bits of transplanted tissue after the first week or ten days, which frequently is the occasion for prompt appearance of growth within another few days, as it seems to stimulate to further development certain lepra bacilli, perhaps the more saprophytic forms in and around the tissue bits. Once the bacilli are accustomed to the new environment, however, there is no difficulty in increasing the rapidity of growth by frequent subculturing. In two of our older cultures the transfers now multiply with great rapidity and reach the maximum. growth in three days. 
Once $B$. lepro has started to grow rapidly, special media such as tryp- . tophan, cystein, etc., are not essential. The culture now does well on any neutral or slightly alkaline medium of human or rabbit blood-agar and glycerinated serum-agars.

Attempts have been made to cultivate the lepra bacilli from nasal secretions in cases in which large numbers of them are found in the serous discharge, and even though they may be contaminated with other bacteria, growth has resulted in two out of five cases in which we have attempted it. The contaminating bacteria, provided there are no spore-bearing varieties present, may be first eliminated by suspending some of the infested secretion in saline solution and heating the suspension at $60 \mathrm{C}$. for thirty minutes. It is much more difficult to obtain cultures of $B$. leprce from nasal discharges than from the tissues, because in the latter, bits of tissue are unavoidably carried over in the transplants, which serve as pabulum for the culture until growth is well started. The same, of course, holds true for other acid-fast species, more especially the tubercle bacillus, whose initial cultivation in many respects is attended with the same difficulties as that of the lepra bacillus.

In a previous paper ${ }^{1}$ on the cultivation of $B$. leproc it was stated that the morphology of the bacillus under artificial growth conditions corresponded closely with that of the organism found in the tissues. This in the main is true, but as two of the original four strains studied have recently undergone a marked change in size, shape and manner of arrangement, we feel that much is yet to be said on the variation in morphology for artificially grown lepra bacilli.

The organisms, when freshly isolated from the animal tissues, are slightly curved and distinctly beaded, a feature that is constant for all early cultures, no matter on what medium they are grown. In some of the older cultures, however, those many generations removed from the parent stem, the bacilli undergo a decided change in size, shape and manner of their arrangement. Not only is the growth more rapid, which naturally would be expected, but the arrangement of the bacilli is diplobacillary, the individual organisms resembling in size the young forms of bovine tubercle bacilli. The difference is so marked that on morphological appearance alone it is quite easy to determine cultures of recent isolation from those that are many generations removed from the animal body. In the first dozen or so subplants from the parent stem the bacilli invariably grow in felt-like masses of slightly curved, slender, beaded rods, while in cultures which are many generations removed they grow diffusely over the medium and occur in pairs of short, ovoid, plump rods. This diplobacillary arrangement of egg-shaped rods is a characteristic feature for the older cultures. In the examination of stained microscopic preparations it is difficult to find single bacilli or clusters of more than two pairs. If such a preparation is stained by any of the ordinary 
anilin dyes it would be hard indeed to distinguish the organism from Diplococcus lanceolatus.

Though this transformation indicates that a given lepra culture is rapidly growing and has become accustomed to a saprophytic existence it is no criterion that the organism has lost its virulence. The same number of hacilli produce lesions in animals as quickly as they did when recently isolated. It is noteworthy in this connection that the bacilli passed through the animal body again become long slender "beaded" rods without a suggestion of pairing, and their recovery from the animal on artificial medium is accompanied with the same difficulty as in the case of the human tissue, growing at first slowly and only on special media.

The staining reaction of the diplo-forms with respect to acid-fastness remains unaltered; in fact they retain more tenaciously the carbol-fuchsin dye than formerly, since they take the stain more intensely and resist for a longer period the decolorizing agents. This form of $B$. leprce multiplies rapidly on a variety of media, good growth occurring in forty-eight hours after incubation at $32 \mathrm{C}$. or $37 \mathrm{C}$. The culture gives the luxuriant growth common to other bacteria that divide as rapidly, and reaches its maximum growth in three days. These older, actively growing cultures still retain their virulence for white and dancing mice.

In some cultures, especially where growth has attained its maximum, many of the diplo-forms of $B$. leproe undergo still another change in morphology. Occasionally in a given culture a great number of the bacilli will show as spindle-shaped rods with swollen centers. This central bulging often appears as an oval clear space with the chromatin densely massed at both poles. These cultures, when heated at $70 \mathrm{C}$. for thirty minutes, are still capable of reproduction. In two of our older cultures this central bulging with the formation of egg-shaped central bodies has repeatedly occurred. Just what factors determine sporulation (assuming that these spaces represent spores) we have not been able to determine, but from analogy, the same factors that influence spore-formation in well-known bacteria do not seem to apply to $B$. lepro, as not all cultures under unfavorable conditions of food, temperature, etc., form "clear spaces." Spore-formation with $B$. leprce might account for successful cultivation after heating at $60 \mathrm{C}$. but, as in the case of tubercle bacilli, the fatty envelope always has to be taken into account, which in itself may sufficiently protect the organism against moderate degrees of heat.

In the cultures that change in morphology the transformation is easily followed. From the granular substance within the bacilli that are lightly stained and distinctly beaded, ovoid masses develop. The chromatin of the parent bacilli seems to undergo amitotic division into two equal parts, which lie end to end in the long diameter of the organism. 
These chromatin masses become more and more definite in outline until two well-formed, deeply staining oval bodies appear in the parent cell. The old cell membrane then undergoes metamorphosis, freeing the pair of oval bodies as a diplobacillus. All forms, from small irregular masses of chromatin to perfect diploid bodies, can be seen in a single field of a preparation of $B$. leprce. There is no mistaking the manner of reproduction of the bacilli during this period of transformation. As soon as the culture has lost all its long beaded forms, however, reproduction then occurs by simple fission. In stained preparations from cultures undergoing this transformation the contrast in density of coloring between the old slender beaded rods and the new oval forms is very striking; the new forms always take the carbol-fuchsin stain more deeply and in consequence decolorize with more difficulty.

Under some conditions, more especially when the organisms are grown in an acid medium or when they are associated with a too profuse growth of symbiotic bacteria, the diplobacillary forms undergo still another change in which one organism in a pair will swell up to twice the size of its fellow and become distinctly spindle-shaped; or both organisms of a pair will simultaneously show the same alteration, forming lancets that are pointed at the distal and flattened at the proximal ends, giving rise to the appearance of certain forms of pseudo-diphtheria bacilli ( $B$. hofmani). These so-called "involution forms" will take place in fortyeight hours when conditions are unfavorable. In old cultures in which growth has ceased, however, they may be kept for months without showing any change in the size and shape of the bacilli. The question of how the administration of different amino-acids, as well as the direct and indirect supply of oxygen influence the growth of parasitic organisms is being studied by one of us (Duval) in collaboration with Dr. Gustav Mann.

\section{IMMUNITY}

Following the cultivation of $B$. lepres our attention was naturally drawn to the possibility of producing an active artificial immunity which might be made use of in the treatment of the disease. In this respect our efforts have been directed along two distinct lines: patients suffering from the disease were inoculated with suspensions containing leprosy bacilli and their toxins; and animals have been inoculated with larger doses of culture in the hope of producing in their serums immune bodies which might be used in the passive immunization of human subjects. In addition an effort has been made to identify the presence of such bodies in the blood from patients suffering from the disease. By means of these qualitative and quantitative tests it was hoped that some circumstantial evidence, at least, might be adduced which would suggest the probable outcome of efforts in serum or vaccine therapy. 
In our first examination four bodies were Iooked for, namely, specific amboceptors, agglutinins and opsonins against the leprosy bacillus and variations in the complement content from the normal and in patients suffering from the disease at its different stages and types. At present we are prepared to report on the complement and amboceptor content in twenty-eight serums from lepers of different types. It has been determined that the complement content does not differ from that in normal individuals to any demonstrable extent, nor does the stage or type of the disease produce any alteration in the quantity of this body present. A paper is now in process of preparation by one of us which will discuss, in extenso, the variations of complement in different diseases, both acute and chronic and in normal individuals; therefore, space will not be taken up here with the discussion of the methods employed nor with a more precise statement of the variations found.

We consider that since complement is present in leprous patients in normal quantities it will probably be of the utmost importance in the treatment of the disease by serum therapy if it is found possible to produce a serum in animals which will contain a high amboceptor content against the Bacillus leprox.

In testing for the specificity as well as the quantity of the amboceptor two antigens were utilized: first, a suspension in saline solution of cultures of the organism grown on tryptophan medium; and secondly the so-called syphilitic antigen consisting of acetone-insoluble lipoids extracted from the human heart by alcohol and ether was employed. Of the twenty-eight serums fifteen were from cases of tubercular types of the disease, seven from anesthetic and six from the so-called mixed type. Although most of the cases showed well-marked and unmistakable evidence of the activity of the disease, few could be classified as advanced and in certain others which will be referred to later there had existed a doubt in the clinician's mind as to the actual presence of the disease in the individuals during the past few years. As is seen from Table 1, the serum from this last group of cases failed to show the presence of specific antibodies. We wish to note here that it was not until the attention of the clinician was drawn to the absence of specific antibodies in those cases that we were informed that the patients were considered free from active disease. In determining the presence of specific antibodies active serum was used, and in reading the results those cases marked $(++)$ represent serums in which at least three quantities of complement are bound. In those marked weakly positive $(-+)$ at least two, and in those marked doubtful ( $( \pm)$ only one quantity is fixed. Before proceeding to a discussion of the results obtained and their significance as interpreted by us, Table 1 will be considered. 
Table 1.-Results of Complement Fixation Tests with leprous Serums

Leprosy Antigen. Lipoid Antigen.

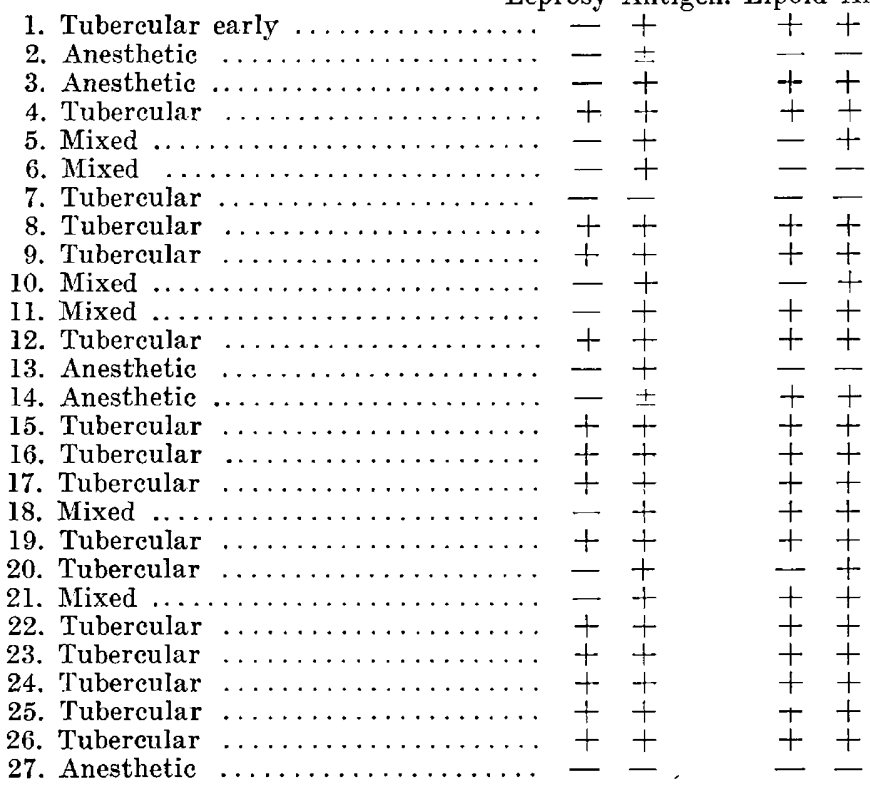

In this table several interesting points are noted; in the first place all but three cases show bodies capable of combining with and fixing complement in the presence of leprosy bacilli and in thirteen cases this binding power is well marked. Of the serums giving a complete reaction with the bacilli, all give a similar reaction in the presence of phosphate containing lipoid substances; and all giving negative results with the organisms are also negative with the phosphatids as an antigen. When the second part of the table is examined, seventeen cases are seen to possess a marked binding power, and eight give negative results. In all instances cases showing no complement-binding power in the presence of the lipoids have been found to possess either little or none when the bacillary emulsion was employed.

To control the reactions with the leprous serums similar reactions were carried out with the blood from patients other than lepers, and at first a most astonishing result was obtained, the significance of which was not at first understood. As controls, serums collected for diagnosis by the Wassermann reaction were utilized, and it was found that a large number of these gave positive results with the bacillary suspension. It was found in the first ten serums tested that six gave a marked binding and two a weak reaction, only two failing to fix any complement whatever. This result was unlooked for, and seemed to upset any inferences whicn might have been drawn from the results of reactions with serums from leprous individuals. It is further noted, however, that of those cases 
fixing complement in the presence of leprosy bacilli all give a marked Wassermann reaction and are undoubtedly syphilitic cases, while one, a tabetic, gave a weak Wassermann reaction.

Table 2.-Results of Complement Fixation in Serums from Cases Other THAN LEPROUS

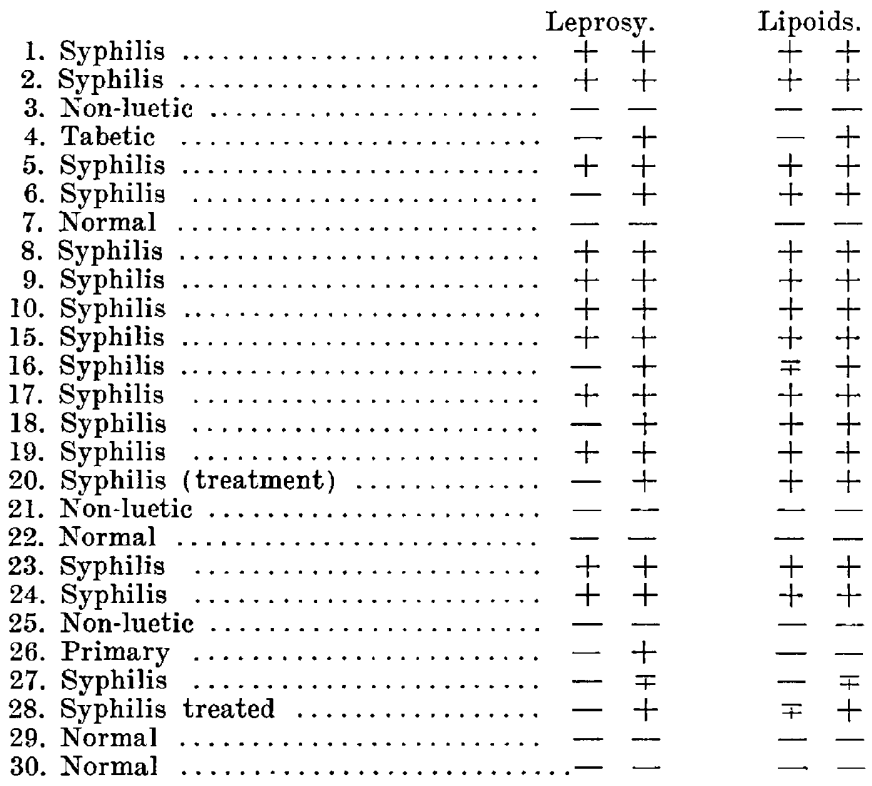

Of the three leprous patients failing to give any reaction with either antigen, two (Numbers 13 and 27 ) are individuals whose history shows that the disease has been present for twenty or twenty-five years and in whom for many years past no progression of the disease has been noted. Both are free from macular or tuberculous lesions and are detained in the home chiefly because the ravages of the disease during the active stage have resulted in so great a deformity that it would be impossible for the patients to go about among society without being subject to criticism. The other patient (No. 7) is a young man in whom all evidence of leprosy has disappeared but who is dying from a severe tuberculous infection. Of the cases giving the weakest reaction all show very little if any evidence of active disease.

Repeated tests prove amply that the suspension of leprosy bacilli used possessed neither anticomplementary powers nor hemolytic properties. There remains, therefore, but one apparent explanation of the phenomenon of cross-reaction between syphilitic and leprous patients with both lipoid bodies and the leprosy bacilli as antigen. Whatever may be the cause of the phenomenon producing antibodies against phosphatids in the blood of syphilitic patients, it is apparent that it is easy to explain 
the presence of antibodies against fatty materials in leprous patients, since it is generally accepted that a fatty or waxy substance forms an important constituent of the leprosy bacillus.

In order, therefore, to prove the presence or absence of specific amboceptors in leprous serums against the other substances in the bacilli, two methods of experimentation were utilized. First, it was endeavored to bind by means of an excess of lipoid body and complement the antibodies against the fatty substances, and then by adding a further content of complement and leprosy bacilli, to bind, if possible, more complement by means of the specific amboceptor against the constituents or toxins of the bacilli other than the fatty substances. In the second series of experiments we attempted to demonstrate the presence of such antibodies by an examination of various serums, especially those in which a small amount of lipoid body was present, carrying out two series of reactions simultaneously in the presence of varying amounts of complement. Up to the present we have been unable to demonstrate by either of these methods specific bodies in leprous serums capable of fixing complement in the presence of products of the bacilli other than the lipoid substances.

In attempting to identify specific agglutinins against the Bacillus lepro, we have found that serums from certain patients at least will agglutinate thoroughly broken-up suspensions of the bacilli in dilutions of 1 to 50 in two hours. Serum from normal individuals has failed to agglutinate bacilli in dilutions of 1 to 10 . We have not found cases from which the serum would agglutinate in dilution of 1 to 100 or more.

So far, we are only in a position to report regarding the immune bodies developed in one experimental animal; namely, a monkey (Macacus rhesus), which was injected at intervals with living leprosy bacilli. Up to the time of examination four doses of a suspension of from 20 to 100 million live lepra bacilli had been injected into the subcutaneous tissue over the chest and in the groin. Following each inoculation a swelling had appeared about the point of inoculation within two or three days which gradually increased in size. The reactions performed on his blood at the end of one month showed that the serum agglutinated the bacilli in dilutions of 1 to 50 , whereas the serum from the normal monkey showed not the slightest tendency toward agglutination. In determining the amboceptor in the monkey's serum it has been found that it binds complement in the presence of phosphatids, even in small quantities, so that further tests are necessary to determine the presence of amboceptors in the serum of this animal.

On October 22, twelve patients were inoculated with a suspension of leprosy bacilli containing eight million organisms to the cubic centimeter. The vaccine was prepared by preliminary heating at $70 \mathrm{C}$. for ten minutes and subsequently the finely divided bacilli were treated in 
a 2 per cent. phenol solution for forty-eight hours. The material was then diluted to six times its volume and used in this manner.

The human cases in which we used the inoculations consisted of moderately advanced tubercular or mixed types together with one long-standing anesthetic case. On the occasion of the first inoculation, doses varying from 0.1 to 0.25 c.c. were used, being given either into the true skin or into the subcutaneous tissue. Following the first administration four patients, each of whom had received 0.2 c.c., reacted locally as evidenced by a small zone of redness about the point of inoculation.

On the second dose (October 29) the quantity injected was increased to from 0.25 c.c. to 4 c.c. without the development of a reaction in any case. Four treatments have so far been given, the dose having been arbitrarily placed at four million bacilli. We cannot, at present, report on any definite progress in the clinical condition of any of these patients, but it may at least be said that no harmful results have ensued.

\section{SUMMARY AND DISCUSSION}

$B$. lepr:s may be cultivated on a variety of artificial media, and are capable of living and retaining their infectibility for months under adverse conditions outside of the animal body. The bacilli can also be cultivated from contaminated leprous tissue and from the nasal discharge that has been kept at room temperature for more than a year. Remarkable as it may seem, it is nevertheless the case that contaminating microorganisms have no appreciable effect on the viability and infectivity of some strains of lepra bacilli.

Pure cultures of $B$. lepra can be obtained directly from the infested leprous tissue on a variety of special media including tryptophan and glycerinated blood-agar without first growing them in the presence of amebas and their symbiotics. From two cases of leprosy we have cultivated in pure growth the specific organism directly from the tissues on Novy-McNeal rabbit blood-agar to which 1 per cent. glycerin had been added. As a rule, multiplication of $B$. leproe on artificial medium takes place slowly, but once the growth has started it can be readily accelerated by frequent transplantation.

At the present writing we have succeeded in cultivating $B$. leprce from the cutaneous nodules in eight cases of leprosy, two cases of nasal discharge, and from the experimental lesion in a number of monkeys and white and Japanese dancing mice. That the cultures are leprosy bacilli, and not some other acid-fast species, has been definitely proved by cultural and animal tests.

The experimental study on the virulence and viability of leprosy bacilli shows the necessity of early diagnosis and the need of strict segregation of certain types of the disease. The length of time B. lepro will live and retain its infectiousness outside the body indicates plainly the 
risk to a community in allowing leper patients at large, in particular those who have open lesions. Especially dangerous from the standpoint of source of infection are the patients discharging the bacilli in the secretions from the nasal mucous membrane. These patients are a constant menace to those with whom they associate because of the possibility of indirect transmission of the bacilli that are unconsciously deposited on articles about the household where the leper resides.

The mere fact that the organism lives for so long a time outside of the animal body may explain why the disease continually reappears in households that have harbored a leper. The bacilli escaping from the infected individual, who for months may not be aware of his malady, are a constant menace to others of the household or to subsequent tenants, even though it be years after the direct source of infection has been removed. The animal experiments do not tend to support the view that leprosy may reside for years in the human body before manifesting any outward signs of the disease.

Direct inoculation from man to man may occur, but it is the exception. On the other hand, in the light of our present knowledge the indirect evidence of transmission is by far the more significant. We believe that this is the most likely condition that renders transmission of the disease possible. It is recognized that other conditions, such as virulence of the bacilli, suitable port of entrance and susceptibility, play an important part, as they do in all infectious diseases. Our investigations also confirm the belief that the mucous membrane of the nasal pharynx is the port through which the bacilli gain entrance to the body, as well as the chief source from which infection spreads.

The results of animal experimentations demonstrate the fact that direct communication of the disease may take place from individual to individual without the presence of the bed-bug or other parasites as intermediate hosts.

On two occasions we have succeeded in infecting mice by rubbing cultures into the nares after gentle scarification of the mucous membrane. These experiments support the view that the chief portal of entrance for $B$. lepres to the human body is by way of the nasopharynx. There is also evidence to show from animal experiments that the bacilli may gain entrance to the human body through breaks in the skin without giving rise to lesions at the entrance site.

In the examination of blood from patients suffering from leprosy the serum has been shown to contain specific antibodies of different kinds against certain constituents of the $B$. leprox. In addition to the presence of specific bodies, we have demonstrated that complement is present in normal quantities. Agglutinins are present, though not in very large amounts. The opsonic content is probably affected at different stages of 
the disease, but with reference to the activity of this body our researches have so far not proved conclusive.

Specific amboceptors are undoubtedly present in comparatively large quantities. In confirmation of the results of others we have found that not only specific bodies are present in the blood of leprous patients binding complement with leprosy bacilli, but also substances capable of fixing this body in the presence of phosphatids prepared by the extraction of human heart muscle. Not only has this phenomenon been constantly present, but it has been demonstrated that the complement-binding power is as great in the presence of the lipoids as in the presence of the bacilli themselves. Thus serums fixing nine units of complement when treated with a suspension of $B$. leprce are likewise found to be capable of fixing a similar quantity when the lecithin antigen is employed; in the same way serums fixing two units with one antigen have fixed two units with the other. That the reaction is quantitatively as well as qualitatively specific is shown by the fact that with few exceptions, even the delicate reaction depending on the fixation of only one complement unit, it has been equal with both materials as antigen.

On the other hand we have been unsuccessful in demonstrating the presence in leprous serums of specific amboceptors other than those combining with the lipoid bodies. Again, we have found that not only is the complement-binding power in the presence of the two antigens a characteristic of leprous cases but it is also found in the serum from luetic individuals. Apparently, then, the constituents of the leprosy bacillus against which the most active antibody is produced is the lipoid material.

Furthermore, we have been successful in extracting from the bacilli by means of ether and alcohol fatty substances in comparatively large quantities, the greater portion of which is insoluble in acetone. This acetone-insoluble content has by analysis been found to contain phosphorus, proving that it belongs to the phosphatid group of fats. Since chemically the fatty material in the bacilli is closely related to the lecithins as extracted from the human heart, and the amboceptor content of the serum of both leprons and luetic cases is quantitatively as well as qualitatively the same whether the artificially prepared lipoids or the bacilli themselves are used, we consider that it is justifiable to assume that these two lipoids can differ but slightly from one another. Furthermore, in view of the fact that the serum of leprous individuals contains chiefly antibodies against the fatty content of the bacterium, and since specific immune bodies are usually produced in a manner in which they will be most useful to the host, we think it reasonable to infer that it is the fatty constituent of the bacillus which offers the greatest protection to the bacilli against the action of the individual's resistant forces.

The idea that the fatty material in the bodies of both tubercle and. leprosy bacilli is a powerful protection against the body fluids and cells 
is by no means new. In fact, in the treatment of leprosy both tuberculin and nastin have been employed in the hope of increasing the resistance of the individual against this protective covering on the bacillus. The absence of satisfactory results following the employment of both these materials can be explained in two ways. First, the fatty constituents of both these preparations differ to a greater extent from that present in the leprosy bacillus than do the phosphatids prepared from cardiac muscle; and secondly, along with the fatty material injected a larger or smaller quantity of toxic bodies other than the lipoids is inoculated, rendering the use of large doses impossible.

We have been sufficiently impressed by the apparent importance of the specific bodies against the phosphatid fat in the immunization against the $B$. lepree to have undertaken animal experimentation in the hope of preparing a serum of sufficient antilipoid potency to be of service in the passive immunization of human cases. Leprous patients are also being subjected to injections of emulsions of the fats to determine the possibility of the production of a more marked active immunity than that produced normally by these cases.

Although we consider that our researches suggest the prime importance of this phase of the immunity leading to the destruction of the bacillus, we believe that this method may probably be supplemented by the production of either a passive or active immunity against the toxins and protein constituents of the bacillus. We have proved the presence of soluble toxins and may assume apparently the presence of endotoxin and toxalbumin, against all of which it should be our aim to develop specific bodies if the greatest advantage in the treatment of the disease is to be achieved. To this end we are continuing the vaccine treatment of cases and are proceeding with the attempt to produce an active immunity in animals. 